\title{
A STATISTICAL TEXTURE FEATURE FOR BUILDING COLLAPSE INFORMATION EXTRACTION OF SAR IMAGE
}

\author{
Linlin Li, Hui Yang, Qihao Chen*, Xiuguo Liu \\ Faculty of Information Engineering, China University of Geosciences (Wuhan), Wuhan 430074, China \\ 1llcug@163.com,yh_cug@163.com, chenqihao@cug.edu.cn, liuxg318@163.com
}

\author{
Commission III, ICWG III/Iva
}

KEY WORDS: Synthetic Aperture Radar (SAR), Building Collapse Information, Texture, $\mathrm{G}^{0}$ Distribution, Texture Parameter

\begin{abstract}
:
Synthetic Aperture Radar (SAR) has become one of the most important ways to extract post-disaster collapsed building information, due to its extreme versatility and almost all-weather, day-and-night working capability, etc. In view of the fact that the inherent statistical distribution of speckle in SAR images is not used to extract collapsed building information, this paper proposed a novel texture feature of statistical models of SAR images to extract the collapsed buildings. In the proposed feature, the texture parameter of $\mathrm{G}^{0}$ distribution from SAR images is used to reflect the uniformity of the target to extract the collapsed building. This feature not only considers the statistical distribution of SAR images, providing more accurate description of the object texture, but also is applied to extract collapsed building information of single-, dual- or full-polarization SAR data. The RADARSAT-2 data of Yushu earthquake which acquired on April 21, 2010 is used to present and analyze the performance of the proposed method. In addition, the applicability of this feature to SAR data with different polarizations is also analysed, which provides decision support for the data selection of collapsed building information extraction.
\end{abstract}

\section{INTRODUCTION}

Accurate collapse information is essential to providing decision support for the government's post-disaster relief and reconstruction. Synthetic Aperture Radar (SAR) has become one of the most effective means of extracting collapsed building information after a disaster, due to its wide coverage, strong penetrability and all-time/all-weather imaging capabilities.

Abundant single-, dual-polarization SAR data provide protection for extracting collapsed buildings information timely after a disaster. The difference between amplitude or backscatter intensity (Ohkura et al, 1997; Matsuoka and Yamazaki, 2005; Hosokawa et al., 2009) and the coherence of (Ito and Hosokawa, 2003; Hosokawa and Jeong, 2007, Liu and Yamazaki, 2015) are often used to extract collapsed building information based on single or dual-polarization SAR images before and after a disaster. In addition, texture features are widely used in the extraction of collapsed building information with single- or dual-polarization SAR data. Analyzing the Cosmo / SkyMed and Terra SAR-X data from Yingxiu town after the Wenchuan earthquake, Dell'Acqua et al. (2009) found that homogeneity in urban areas decreased as building damage increased. Polli et al. (2010) calculated the correlations between the earthquake damage level and the eight texture based on the Gray Level Co-occurrence Matrix (GLCM). The results showed that entropy, homogeneity and earthquake damage level had a relatively good correlation. Based on the homogeneity and entropy of GLCM, Dell'Acqua et al. (2010) classified the damage levels into three levels and experiment with postearthquake single-polarization SAR data from the 2008 Wenchuan, the 2009 L'Aquila Italy and the 2010 Haiti earthquake, although the extent of damage for some blocks are overestimated, the follow-up study is still of great significance.

\footnotetext{
* Corresponding author
}

Compared with the single- and dual-polarization SAR, fullpolarization SAR (PolSAR) obtains richer surface information. Polarization features have been widely used in collapsed building extraction of PolSAR image, and has achieved good results (Guo, 2009; Li et al., 2012; Zhao et al., 2013; Shen et al., 2015). But it is easy to misidentify the intact buildings with large orientation angle as collapsed buildings because of the influence of orientation angle and building structure. Zhao et al. (2013) revised the results of building damage assessment, brought about by normalized circular-pol correlation coefficient (NCCC), with the help of the homogeneity texture features of the GLCM. Shi et al. (2015) extracted 181 features of polarizations, interferences and textures to classify airborne SAR data after Yushu Earthquake and evaluated building damage by random forest classifiers. The result proved that texture features were superior than polarization feature in extracting collapsed buildings, and the variance of Gray-Level Histogram extracted based on X-band intensity data was best. Sun et al. (2016) extracted 5 categories of texture features based on the CASMSAR data of the urban area after the Yushu earthquake. Random forest classifier was used to evaluate the damage levels of buildings. The test indicated the variance of the Gray-Level Histogram and the contrast texture based on GLCM worked well in assessment of collapsed buildings generally.

Texture features play an important part in assessment of collapsed buildings in SAR data. But directly applying the extraction method of optical images to SAR images can't accurately describe the texture information of the object. Owing to it does not consider the inherent statistical distribution of speckle in SAR images. Studying the statistical characteristics of speckle in SAR images can better extract the information needed. In order to better model the extremely heterogeneous regions in urban areas, Frery et al. (2008) modelled the texture variables with inverse gamma distribution and proposed a $\mathrm{G}^{0}$ distribution suitable for the modelling of extreme heterogeneous 
regions, which is based on the product model. The texture parameter of the $\mathrm{G}^{0}$ distribution can reflect the uniformity of the target. The larger the value of the texture parameter, the more uniform the target (Frery et al., 2008; Lee and E. Pottier, 2009).

Therefore, in this paper, the texture parameter of $\mathrm{G}^{0}$ distribution in SAR image is used to describe the uniformity of the target, so as to extract the information of collapsed buildings. In addition, this paper also comparatively analyses the applicability of $\mathrm{G}^{0}$ texture parameter to SAR image with different polarization in extracting collapsed building information, and provides the basis for the post-disaster collapsed building information extraction in SAR images. The experiments of the RADARSAT-2 data after the Yushu earthquake in 2010 prove the validity of statistical texture features proposed in this paper for extracting collapsed buildings information.

\section{METHODOLOGY}

The process of this method includes three main steps. Firstly, the $G^{0}$ texture parameter is estimated based on the SAR data. Then, the non-building areas are removed with the method of literature (Chen et al., 2016), and the collapsed buildings are extracted utilizing the estimated texture parameter. Finally, the build damage assessment map is obtained according to the building block collapse rate (BBCR). The detailed flow chart is shown in Figure 1.

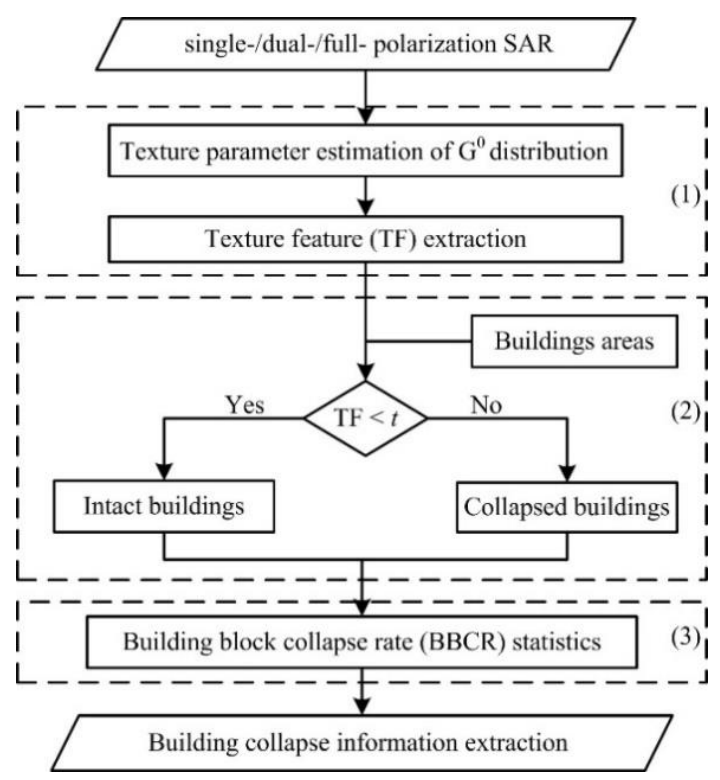

Figure 1. The flow chart of building damage assessment.

\subsection{SAR data}

In a reciprocal medium, the full-polarization SAR scattering vector is $u=\left[\begin{array}{lll}S_{H H} & \sqrt{2} S_{H V} & S_{V V}\end{array}\right]^{T}$, the covariance matrix is

$$
\begin{aligned}
\mathbf{C}_{3} & =\left\langle u \cdot u^{* T}\right\rangle \\
& =\left[\begin{array}{ccc}
\left\langle\left|S_{\mathrm{HH}}\right|^{2}\right\rangle & \sqrt{2}\left\langle S_{\mathrm{HH}} S_{\mathrm{HV}}{ }^{*}\right\rangle & \left\langle S_{\mathrm{HH}} S_{\mathrm{VV}}{ }^{*}\right\rangle \\
\sqrt{2}\left\langle S_{\mathrm{HV}} S_{\mathrm{HH}}{ }^{*}\right\rangle & 2\left\langle\left|S_{\mathrm{HV}}\right|^{2}\right\rangle & \sqrt{2}\left\langle S_{\mathrm{HV}} S_{\mathrm{VV}}{ }^{*}\right\rangle \\
\left\langle S_{\mathrm{VV}} S_{\mathrm{HH}}{ }^{*}\right\rangle & \sqrt{2}\left\langle S_{\mathrm{VV}} S_{\mathrm{HV}}{ }^{*}\right\rangle & \left\langle\left|S_{\mathrm{VV}}\right|^{2}\right\rangle
\end{array}\right]
\end{aligned}
$$

where $T$ is the transpose of the matrix,

* indicates the conjugate,

$\langle\cdot\rangle$ represents the set average,

$\sqrt{2}$ is to ensure that the total power is equal.
Scattering vectors for dual-polarization SAR data can be written directly as $u=\left[\begin{array}{ll}S_{1} & S_{2}\end{array}\right]^{T}$, the polarization covariance matrix is:

$$
\mathbf{C}_{2}=\left\langle u \cdot u^{* T}\right\rangle=\left[\begin{array}{ll}
\left\langle\left|S_{1}\right|^{2}\right\rangle & \left\langle S_{1} S_{2}{ }^{*}\right\rangle \\
\left\langle S_{2} S_{1}{ }^{*}\right\rangle & \left\langle\left|S_{2}\right|^{2}\right\rangle
\end{array}\right]
$$

For the HH / HV combination in dual polarization SAR image, the polarization covariance matrix is:

$$
\mathbf{C}_{2}=\left[\begin{array}{cc}
\left\langle\left|S_{\mathrm{HH}}\right|^{2}\right\rangle & \left\langle S_{\mathrm{HH}} S_{\mathrm{HV}}{ }^{*}\right\rangle \\
\left\langle S_{\mathrm{HV}} S_{\mathrm{HH}}{ }^{*}\right\rangle & \left\langle\left|S_{\mathrm{HV}}\right|^{2}\right\rangle
\end{array}\right]
$$

For the HV/VV combination in dual polarization SAR image, the polarization covariance matrix is:

$$
\mathbf{C}_{2}=\left[\begin{array}{cc}
\left\langle\left|S_{\mathrm{HV}}\right|^{2}\right\rangle & \left\langle S_{\mathrm{HV}} S_{\mathrm{VV}}{ }^{*}\right\rangle \\
\left\langle S_{\mathrm{VV}} S_{\mathrm{HV}}{ }^{*}\right\rangle & \left\langle\left|S_{\mathrm{VV}}\right|^{2}\right\rangle
\end{array}\right]
$$

For the HH/VV combination in dual polarization SAR image, the polarization covariance matrix is:

$$
\mathbf{C}_{2}=\left[\begin{array}{cc}
\left\langle\left|S_{\mathrm{HH}}\right|^{2}\right\rangle & \left\langle S_{\mathrm{HH}} S_{\mathrm{VV}}{ }^{*}\right\rangle \\
\left\langle S_{\mathrm{VV}} S_{\mathrm{HH}}{ }^{*}\right\rangle & \left\langle\left|S_{\mathrm{VV}}\right|^{2}\right\rangle
\end{array}\right]
$$

\section{2 $\mathrm{G}^{0}$ distribution and Parameters Estimation}

According to product model, the speckle of SAR data is expressed as the product of texture variable $\tau$ and speckle distribution $\mathbf{X}$.

$$
\mathbf{Y}=\tau \mathbf{X}
$$

For multi-look and multi-polarization SAR data, $\mathbf{Y}$ is covariance matrix , $\mathbf{X}$ obeys the Wishart distribution, and the PDF (Probability Density Function) is:

$$
\begin{gathered}
W_{d}(\mathbf{X})=\frac{n^{n d}|\mathbf{X}|^{n-d}}{\Gamma_{d}(n)|\mathbf{\Sigma}|^{n}} \exp \left[-n \operatorname{tr}\left(\boldsymbol{\Sigma}^{-1} \mathbf{X}\right)\right] \\
\Gamma_{d}(n)=\pi^{\frac{d(d-1)}{2}} \Gamma(n) \ldots \Gamma(n-d+1)
\end{gathered}
$$

where $\boldsymbol{\Sigma}$ is the expectation of the covariance matrix, $n$ is the number of looks,

$\Gamma(\cdot)$ denotes the gamma function,

$\operatorname{tr}(\cdot)$ represents the trace of the matrix,

$d$ is the dimension of speckle vector, and $d$ is 2 in dual-polarization SAR data. In the reciprocal medium, $d=3$ for full polarization SAR data.

For multi-look and single-polarization SAR data, $\mathbf{Y}$ indicates intensity, The probability density function of $\mathbf{X}$ is a special case of $d=1$ in equation ( 7 ) and can be expressed as :

$$
p(\mathbf{X})=\frac{n^{n} \mathbf{X}^{n-1}}{\Gamma(n) \mu^{n}} \exp [-n \mathbf{X} / \mu]
$$

where $\mu$ is the expectation.

When the texture variable $\tau$ is modelled by inverse gamma $\left(\bar{\gamma}^{-1}\right)$ distribution,

$$
\bar{\gamma}^{-1}(\lambda)=\frac{(\lambda-1)^{\lambda}}{\Gamma(\lambda)} \frac{1}{\tau^{\lambda+1}} \exp \left(-\frac{\lambda-1}{\tau}\right)
$$

where $\lambda$ is the texture parameter. 
For multi-look and multi-polarization SAR data, covariance matrix follows the $G^{0}$ distribution, and the probability density function (PDF) is expressed as:

$$
\begin{aligned}
& p(\boldsymbol{C})=\frac{n^{d L}|\boldsymbol{C}|^{n-d} \Gamma(d n+\lambda)(\lambda-1)^{\lambda}}{\Gamma_{d}(n)|\boldsymbol{\Sigma}|^{L} \Gamma(\lambda)} \\
& \times\left(n t r\left(\boldsymbol{\Sigma}^{-1} \boldsymbol{C}\right)+\lambda-1\right)^{-\lambda-d n}
\end{aligned}
$$

When $d=1$, equation (11) can be simplified to the $\mathrm{G}^{0}$ distribution of the multi-look and single-polarization SAR intensity image I. Its probability density function is:

$$
p(\mathbf{I})=\frac{n^{n} \Gamma(n+\lambda)(\lambda-1)^{\lambda} \mathbf{I}^{n-1}}{\Gamma(n) \Gamma(\lambda)(n \mathbf{I}+\lambda-1)^{\lambda+n}}
$$

Based on the second moment, the texture parameter of the $G^{0}$ distribution is expressed as:

$$
\hat{\lambda}_{D}=\frac{2 n \operatorname{Var}\{M\}+d(n d-1)}{n \operatorname{Var}\{M\}-d}
$$

where $\operatorname{Var}\{\cdot\}$ represents variance, for the multi-polarization SAR data, $M=\operatorname{tr}\left(\Sigma^{-1} C\right)$, for the single-polarization SAR data, $M$ is intensity data.

The texture parameter of $\mathrm{G}^{0}$ distribution reflect the homogeneity of the targets (Frery et al., 2008; Lee and E. Pottier, 2009). The larger the estimated texture parameter is, the smaller the variance of the texture variables become. Therefore, this paper proposes an effective way to distinguish between intact buildings and collapsed buildings using the texture parameters of statistical model.

\subsection{Building Damage Assessment}

Based on the estimated texture parameter, the collapsed buildings are extracted with the threshold which is determined by iterative method.

The building block collapse rate (BBCR) is calculated at the block scale. These blocks are separated by roads, and the

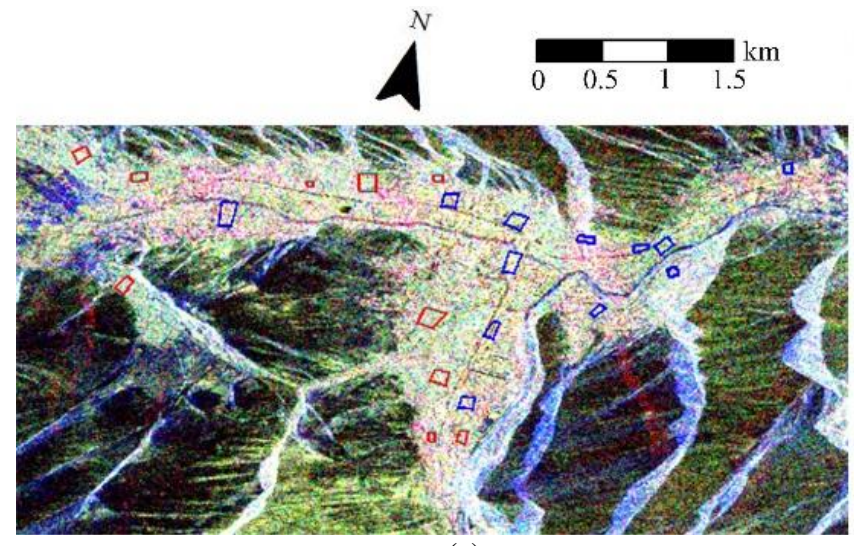

(a) structures of building have a strong similarity in one block (Zhao et al., 2013). BBCR is defined as the proportion of collapsed buildings to the total number of buildings (contains intact buildings and collapsed buildings) of the block. The more serious damage the block suffers, the greater BBCR is. The $\mathrm{BBCR}$ is expressed as:

$$
B B C R_{j}=\frac{N C_{j}}{N I_{j}+N C_{j}}
$$

where $N C_{j}$ and $N I_{j}$ indicate the number of collapsed and intact buildings of the $j$ th block.

The thresholds 0.3 and 0.5 are set based on BBCR, and the blocks are divided into three levels: slight damage, moderate damage and serious damage.

\section{EXPERIMENTAL RESULTS}

\subsection{Description of the Experimental Data}

The study case is Yushu County in the Qinghai Province of China after a 7.1-magnitude earthquake that occurred on April 14, 2010. The epicenter was at $33.1^{\circ} \mathrm{N}$ and $96.7^{\circ} \mathrm{E}$. The elevation of Yushu County is approximately $3500 \mathrm{~m}$, but its terrain is comparatively gentle. The major land cover classes include bare soil, buildings, roads, rivers, and so on. The buildings are mainly rural residential buildings; most do not design to resist seismic events, and some buildings are on slopes or other unstable places. The $\mathrm{C}$ band, RADARSAT-2 polarimetric data has a spatial resolution about $8 \mathrm{~m}$. The Pauli RGB image after 3-look multi-looking processing is shown in Figure. 2(a) at a size of $527 \times 257$ pixels, the areas marked in red are the collapsed buildings; blue indicates intact buildings .

The ground-truth map of the buildings in Yushu County is shown in Fig. 2(b), which was interpreted according to the 0.5 m optical image acquired on May 6, 2010 and the detailed damage map from the German Aerospace Center (DLR, 2016). The whole county is divided into 83 blocks according to roads and the similarities of built-up patches, including slight damage, moderate damage and serious damage, and the classification rule for damage levels are refer to other studies (Zhao et al., 2013; Zhai et al., 2016).

\section{Central Latitude: $32^{\circ} 59^{\prime} 49^{\prime} \mathrm{N}$}

Central Longitude: $97^{\circ} 00^{\prime} 16^{\prime \prime} \mathrm{E}$

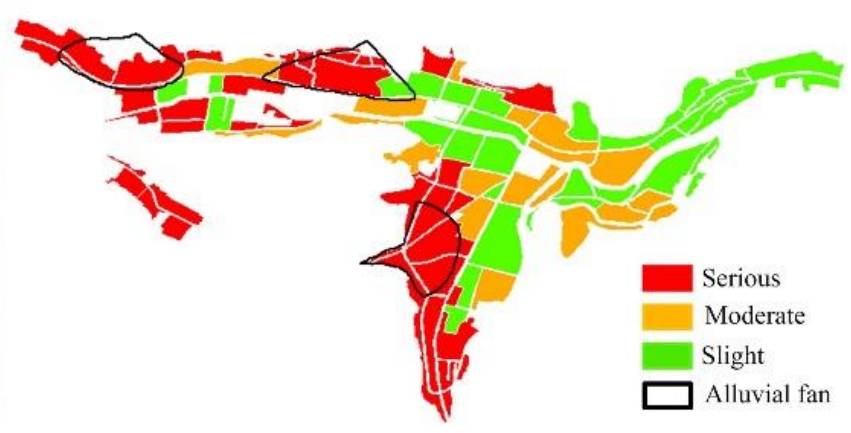

(b)

Figure 2. The C band, RARDRSAT-2 data of Yushu County: (a) Pauli RGB image; (b) Reference map. 


\subsection{Experiment and analysis}

In this paper, the $\mathrm{G}^{0}$ distribution texture parameters of single-, dual- and full-polarization SAR data are calculated by equation (13), which also extracts the texture features to reflect the uniformity of the building area. Using the area under the receiver operating characteristic curve (AUC) (Hanley, 1982) assesses the distinguishing ability of the $\mathrm{G}^{0}$ distribution texture feature $\left(\mathrm{G}^{0}\right.$-para) for collapsed buildings and intact buildings. Contrast texture of Gray Level Co-occurrence Matrix (GLCM) and the variance of the gray histogram (Var-his) were compared to illustrate the effectiveness of the texture features introduced in this paper. Based on the sample in Figure 2(a), the calculated AUC for different features is shown in Table 1.

\begin{tabular}{llll}
\hline & VV & HH/HV & PolSAR \\
\hline Contrast & 0.7789 & 0.8174 & 0.8555 \\
Var-his & 0.8137 & 0.8031 & 0.8191 \\
$\mathrm{G}^{0}$-para & 0.8900 & 0.8919 & 0.916 \\
\hline
\end{tabular}

Table 1. The AUC of $\mathrm{G}^{0}$ distribution texture parameters in SAR data with different polarizations

The AUC reflects the ability of the feature to recognize the sample, independent of the threshold. The larger the AUC, the stronger the ability of the feature to recognize the sample. As shown in Table 1, regardless of $\mathrm{VV}, \mathrm{HH} / \mathrm{HV}$, or fullpolarization SAR data, the recognition ability for collapsed buildings of $\mathrm{G}^{0}$ texture parameter is significantly better than the contrast features.

Statistical texture features extracted from PolSAR data based on VV, HH / HV are shown in Figure.3. Comparing Figure 3 (a) (c) with the reference figure, it can be seen that the value of severely damaged area is significantly larger than that of lightly damaged area, which is consistent with the theoretical analysis in Section 2.2.

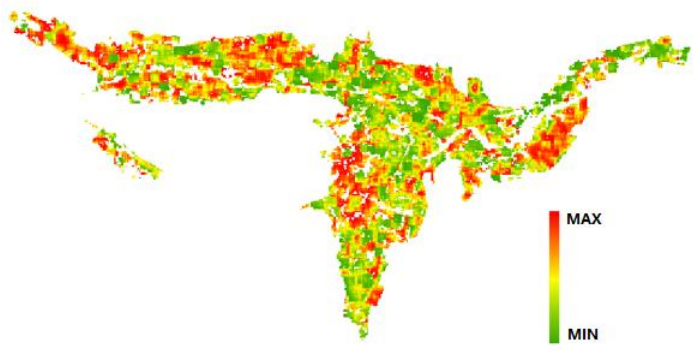

(a)

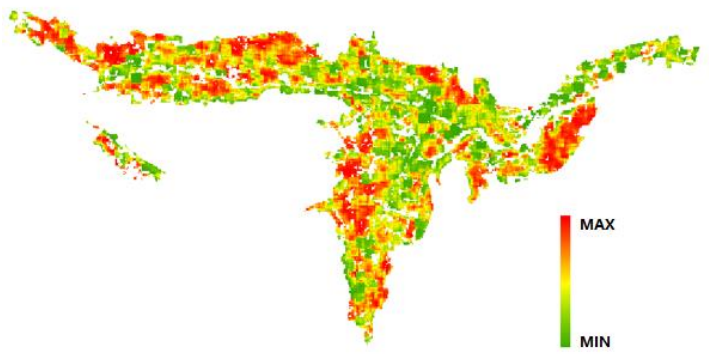

(b)

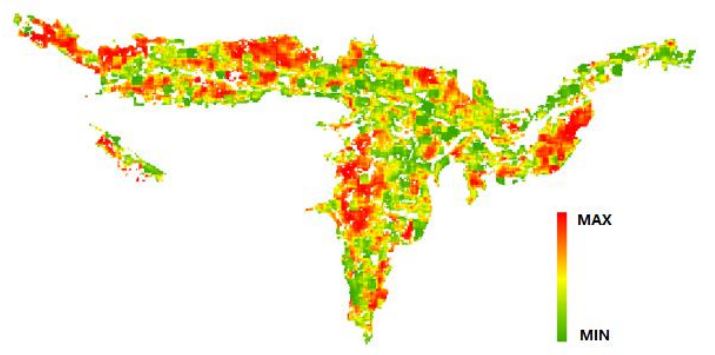

(c)

Figure 3. the display of $\mathrm{G}^{0}$ texture parameter features: (a) VV; (b) HH/HV; (c) Full-polarization SAR.
In order to further demonstrate the effectiveness of the proposed features in collapsed building information extraction, the level of building damage in the Yushu area is assessed according to the method in Section 2.3. the results are shown in Figure 4.

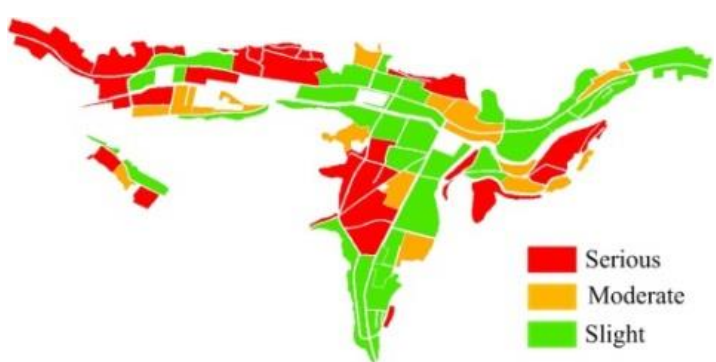

(a)

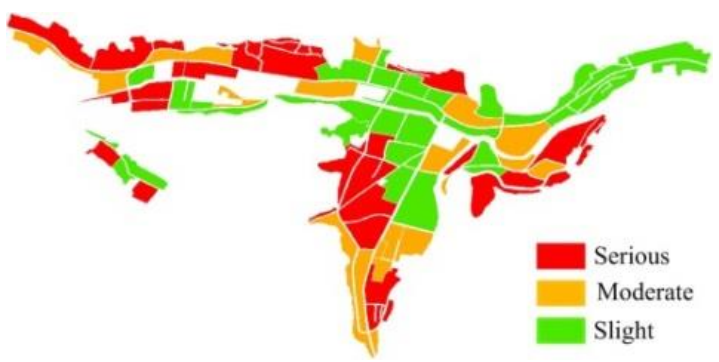

(b)

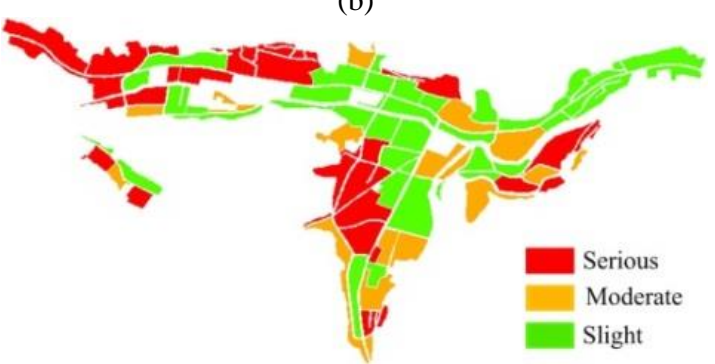

(c)

Figure 4. The building collapse assessment map: (a) VV; (b) $\mathrm{HH} / \mathrm{HV}$; (c) Full-polarization SAR

Basically, the severe damaged areas on the three alluvial fans and the mildly damaged areas on the northeast corner with orientation angles can be accurately evaluated. However, buildings in southeastern of the urban are sparse and uniform, so that mild or moderately damaged areas are easily misjudged as severe damage. Due to the existence of partially intact buildings in the south severe damaged area, the uniformity of the buildings is reduced and is easily misclassified as moderate 
or mild damage. The assessment of VV in the south severe damaged area is worst. Although the assessment of $\mathrm{HH} / \mathrm{HV}$ in the south is more accurate, it is easy to identify some of the severe damaged area in northwest as moderately damaged. In order to quantitatively analyze the effect of building damage assessment, the detection rate (DR), false alarm rate (FAR), F1- score (Powers and Ailab, 2011) and overall accuracy (OA) are considered to evaluate the accuracy as shown in Table 2 . The accuracy of PolSAR is better than that of $\mathrm{HH} / \mathrm{HV}$, which is $68.01 \%, 71.51 \%$ and $73.70 \%$ respectively.

\begin{tabular}{cccc|ccc|ccc|c}
\hline & \multicolumn{3}{c|}{ SE(\%) } & \multicolumn{3}{c|}{ MO(\%) } & \multicolumn{3}{c|}{ SL(\%) } & \multirow{2}{*}{ OA(\%) } \\
\cline { 2 - 9 } & DR & FAR & F1 & DR & FAR & F1 & DR & FAR & F1 & \\
\hline VV & 72.95 & 17.16 & 77.58 & 39.63 & 40.14 & 47.69 & 82.06 & 41.08 & 68.59 & 68.01 \\
HH/HV & 74.43 & 19.64 & 77.28 & 51.39 & 47.42 & 51.98 & 82.22 & 25.90 & 77.95 & 71.51 \\
PolSAR & 75.14 & 12.01 & 81.06 & 51.69 & 33.56 & 58.15 & 87.62 & 34.78 & 74.77 & 73.70 \\
\hline
\end{tabular}

Table 2. The accuracies of building damage assessments for RADARSAT-2 data.

The $\mathrm{G}^{0}$ texture parameters of SAR data with different polarizations are shown in Table 3 , which are based on the sample table 2 in Figure. 2 (a). For single-polarization SAR data, the AUC values of the $\mathrm{G}^{0}$ texture parameters of the samepolarization SAR (HH or VV) are not much different but both larger than HV. In dual-polarization SAR data, the AUC values of the combination of co-polarization and cross-polarization $(\mathrm{HH} / \mathrm{HV}$ or $\mathrm{VH} / \mathrm{VV})$ are all greater than $\mathrm{HH} / \mathrm{VV}$, and the value of $\mathrm{HH} / \mathrm{VV}$ is smaller than $\mathrm{HH}$ or VV. Table 3 shows that the $\mathrm{G}^{0}$ texture parameter of $\mathrm{HV}$ is not suitable for information extraction of collapsed buildings. For single-polarization SAR image, the introduced texture feature of $\mathrm{HH}$ and $\mathrm{VV}$ are similar, and they are better than HV. For dual-polarization SAR image, $\mathrm{HH} / \mathrm{VV}, \mathrm{HH} / \mathrm{HV}$ and $\mathrm{VV} / \mathrm{VH}$ have the similar results, which are close to full-polarization SAR image.

\begin{tabular}{llllllll}
\hline & HH & VV & HV & HH/VV & HH/HV & VH/VV & PolSAR \\
\hline AUC & 0.8833 & 0.8900 & 0.8232 & 0.8631 & 0.8919 & 0.9033 & 0.9160 \\
\hline
\end{tabular}

Table 3. The AUC of $\mathrm{G}^{0}$ distribution texture parameters in SAR data with different polarizations

\section{CONCLUSIONS}

This study introduced the $G^{0}$ distribution texture parameter to reflect the homogeneity of building areas, and it was used for collapsed building information extraction. The introduced statistical texture feature can be applied to single-, dual- and full-polarization SAR images. According to the experiments of RADARSAT-2 SAR image after Yushu earthquake, the texture features proposed in this paper is superior to the comparison features in distinguishing collapsed and intact buildings, and achieves higher accuracy for collapsed building information extraction. When full-polarization SAR image is difficult to obtain, the $\mathrm{G}^{0}$ distribution texture parameters of dualpolarization, $\mathrm{HH}$ and VV SAR images are the suitable choice for collapsed building information extraction.

\section{ACKNOWLEDGMENT}

This work was supported in part by the National Natural Science Foundation of China under Grant No. 41771467 and 41471355 .

\section{REFERENCES}

Bombrun, L. and J.M. Beaulieu, 2008. Fisher Distribution for Texture Modeling of Polarimetric SAR Data. IEEE Geoscience \& Remote Sensing Letters, 5(3), pp. 512-516.

Chen, Q., Li, L., Jiang, P., Liu, X., 2016. Building collapse extraction using modified freeman decomposition from postdisaster polarimetric SAR image. In: IEEE International Geoscience and Remote Sensing Symposium (IGARSS), pp. 5769-5772.
Dell'Acqua F, Lisini G, Gamba P, 2010. Experiences in optical and SAR imagery analysis for damage assessment in the Wuhan, may 2008 earthquake. In: IEEE International Geoscience and Remote Sensing Symposium (IGARSS), pp.37-40.

Dell'Acqua F, Gamba P, Polli D A.,2010. Earthquake damage assessment from post-event VHR radar data: from Sichuan, 2008 to Haiti, 2010.In: Urban Remote Sensing Event, pp.201204.

DLR. Detailed damage assessment map Gyegu-China, Yushu prefecture. Available online: https://www.zki.dlr.de/map/1338 (accessed on 20 April 2016).

Guo H.,2009. Study of detecting method with advanced airborne and spaceborne synthetic aperture radar data for collapsed urban buildings from the Wenchuan earthquake. Journal of Applied Remote Sensing, 3(1), pp.131-136.

Hanley, J. A., Mcneil, B. J., 1982. The meaning and use of the area under a receiver operating characteristic (ROC) curve. Radiology, 143 (1), pp. 29-36.

Hosokawa, M., Jeong, B.P., 2007. Earthquake damage detection using remote sensing data. In: IEEE International Geoscience and Remote Sensing Symposium (IGARSS), pp. 2989-2991.

Hosokawa, M., Jeong, B.P., Takizawa, O., 2009. Earthquake intensity estimation and damage detection using remote sensing data for global rescue operations. In: IEEE International Geoscience and Remote Sensing Symposium (IGARSS), pp. II420-II-423. 
Ito, Y., Hosokawa, M., 2003. A degree-of-damage estimation model of earthquake damage using interferometric SAR data. Electrical Engineering in Japan, 143 (3), pp. 49-57.

Lee, J.S. and E. Pottier, 2009. Polarimetric Radar Imaging: From Basics to Applications. Francis Group Boca Raton Isbn.

Liu W, Yamazaki F,2015. Damage detection in the 2015 Nepal earthquake using ALOS-2 satellite SAR imagery. In: The Pacific Conference on Earthquake Engineering.

Li X, Guo H, Zhang L, et al.,2012. A New Approach to collapsed building extraction using RADARSAT-2 polarimetric SAR imagery, IEEE Geoscience \& Remote Sensing Letters, 9(4), pp.677-681.

Matsuoka, M., Yamazaki, F., 2005. Building damage mapping of the 2003 Bam, Iran, earthquake using Envisat/ASAR intensity imagery. Earthquake Spectra, 21 (S1), pp.285-294.

Ohkura, H., Jitsufuchi, T., Matsumoto, T., Fujinawa, Y., 1997. Application of SAR data to monitoring of earthquake disaster. Adv. Space. Res., 19 (9), pp.1429-1436.

Polli D A, 'Acqua F D, Lisini G., 2010. Automatic mapping of earthquake damage using post-event radar satellite data: the Story Goes On. Remote Sensing for Science, Education and Natural and Cultural Heritage, pp.565-572.

Powers, D.M.W., Ailab, 2011. Evaluation: From precision, recall and F-measure to ROC, informedness, markedness \& correlation. Journal of Machine Learning Technologies,2(1), pp. 2229-3981.

Shen, J. C. and X. Xu, et al., 2015. Collapsed building extraction from single full polarimetric SAR image after earthquake. Science Technology \& Engineering, 15, pp. 86-91.

Shi L, Sun W, Yang J, et al., 2015. Building collapse assessment by the use of postearthquake chinese VHR airborne SAR. IEEE Geoscience \& Remote Sensing Letters, 12(10), pp.2021-2025.

Sun W, Shi L, Yang J, et al., 2016.Building collapse assessment in urban areas using texture information from postevent SAR data. IEEE Journal of Selected Topics in Applied Earth Observations \& Remote Sensing, 9(8), pp.3792-3808.

Zhai, W., Shen, H., Huang, C., Pei, W., 2016. Building earthquake damage information extraction from a single postearthquake PolSAR image. Remote Sensing, 8 (3), pp.171.

Zhao L, Yang J, Li P, et al., 2013. Damage assessment in urban areas using post-earthquake airborne PolSAR imagery. International Journal of Remote Sensing, 34(24), pp.8952-8966. 